\title{
Análisis del fracaso de las MiPyME franquiciantes en México. Un crecimiento cuestionable del sector
}

\author{
MARÍA DEL ROSARIO CORTES-CASTILLO* \\ MARÍA LUISA SAAVEDRA GARCÍA** \\ PABLO DANIEL PALACIOS DUARTE***
}

* Doctora en Planeación Estratégica y Dirección de Tecnología. Escuela Superior de Comercio y Administración del Instituto Politécnico Nacional, Ciudad de México, México. E-mail: mrcortes@ipn.mx. ORCID: 000o-0002-0931-620X. Google Scholar: https://scholar.google. com/citations?user=hmFPyHoAAAAJ\&hl=en.

** Doctora en Administración. Universidad Nacional Autónoma de México, Ciudad de México, México. E-mail: Isaavedra@fca.unam.mx. ORCID: 0000-0002-3297-1157. Google Scholar: https://scholar.google.com.mx/citations?user=iyFudZkAAAAJ\&hl=es.

$\star \star \star$ Doctor en Economía de la Empresa.Benemérita Universidad Autónoma de Puebla, Puebla, México.E-mail:pablo.palacios@correo.buap.mx. ORCID: 0000-0003-3741-770X. Google Scholar: https://scholar.google.com.mx/citations?user=0yKMgvYAAAAJ\&hl=es. 
COMO CITAR ESTE ARTÍCULO

How to cite this article:

Cortes-Castillo, M., Saavedra, M.L. y Palacios, P.D. (2020). Análisis del fracaso de las MiPyME franquiciantes en México. Un crecimiento cuestionable del sector. Revista Perspectiva Empresarial, 7(2), 36-52.

Recibido: 08 de agosto de 2020 Aceptado: 06 de noviembre de 2020
RESUMEN El objetivo de esta investigación consiste en determinar la tasa del fracaso de las MiPyME franquiciantes mexicanas durante el período 2005-2014. Se realizó una investigación exploratoria descriptiva bajo un diseño de investigación longitudinal, que abarco 10 años, ex post facto evolutivo; se recolectaron datos de fuentes secundarias y se estudió la evolución de 7037 Mipymes franquiciantes mexicanas de los sectores comercio y servicios. Entre los principales hallazgos se encontró una caída vertiginosa de las Mipymes franquiciantes mexicanas, las cuales fracasaron en su intento por adoptar este modelo en un $11 \%$; mientras que un porcentaje superior ( $13 \%$ ) abandonó el modelo de franquicias. En conclusión, estos resultados ponen en duda los códigos establecidos de que una franquicia reduce los riesgos de fracaso y facilita la expansión y el crecimiento de una MiPyME.

PALABRAS CLAVE Mipymes, fracaso, franquiciantes, modelo de franquicias, México.

\section{Analysis of the failure of franchising MSMEs in Mexico. A questionable growth of the sector}

ABSTRACT The aim of this research is to determine the failure rate of Mexican franchising MSMEs during the period 2005-2014. A descriptive exploratory research work was conducted under a longitudinal research design, ex post facto evolutionary, covering 10 years. Data were collected from secondary sources and the evolution of 7037 Mexican franchising MSMEs of the commercial and service sectors was studied. Among the main findings was a drastic fall of Mexican franchising MSMEs, $11 \%$ of which failed in their attempt to adopt this model; while a higher percentage (13\%) abandoned the franchising model. In conclusion, these results question the established codes that a franchise reduces the risks of failure and facilitates the expansion and growth of a MSME.

KEYWORDS MSMEs, failure, franchisors, franchise model, Mexico. 


\section{Análise do insucesso das MPMEs franqueadoras no México. Um crescimento questionável do setor}

RESUMO 0 objetivo desta pesquisa é determinar a taxa de insucesso das MPMEs mexicanas de franquia durante o período de 2005-2014. Realizou-se uma pesquisa exploratória descritiva sob um desenho de pesquisa longitudinal, que abrangeu 10 anos, ex post facto evolutivo; os dados foram coletados de fontes secundárias e a evolução de 7.037 MPMEs mexicanas em franquias nos setores de comércio e serviços foram estudadas. Entre as principais descobertas estava uma queda vertiginosa das MPMEs mexicanas em franquia, que falharam em sua tentativa de adotar esse modelo em $11 \%$; enquanto um percentual maior (13\%) abandonou o modelo de franquia. Em conclusão, esses resultados questionam os códigos estabelecidos de que uma franquia reduz os riscos de insucesso e facilita a expansão e crescimento de uma MPME.

PALAVRAS CHAVE MPMEs, fracasso, franqueadores, modelo de franquia, México. 


\section{Introducción}

El modelo de franquicias facilita la creación de nuevas empresas, el aumento del empleo, un mayor acceso a la mujer al mundo empresarial (Díez y Rondán, 2004), así como el incremento de la base tributaria. Al ser un modelo utilizado principalmente por las Mipymes como estrategia de expansión y crecimiento (WFC, 2013) distinguiéndose entre otras formas de organización por el crecimiento acelerado en puntos de venta (Michael, 2003), el alto componente de servicio, una asignación única de responsabilidades, derechos y beneficios a través de un contrato entre los involucrados (Beere, 2017), el financiamiento con el recurso de terceros (franquiciatarios), el conocimiento del inversionista en el mercado local y el acceso en mercados extranjeros (Combs et al., 2011).

Diversas investigaciones han expresado una consolidación de este sector en México tras identificar una tasa de crecimiento de más del $200 \%$ en un lapso de 10 años (Cortes-Castillo, 2019), en parte impulsado por los programas de gobierno enfocados a este sector durante los últimos dos sexenios (2006-2012 y 2012-2018).

A pesar de su importancia existe escasez de investigaciones científicas que aborden el fracaso de las empresas franquiciantes en México ante la ausencia de un organismo que facilite información veraz, relativa y confiable respecto a la evolución del sector (Guerrero, Armenteros y Medina, 2014), así como la tasa del fracaso del franquiciante (Jiménez, 2015). Los franquiciantes en las Mipymes se caracterizan por presentar diversas áreas de oportunidad en la gestión (ENAPROCE, 2018), siendo los sectores comercio y servicios los que cuentan con el mayor índice de mortalidad y nacimiento (INEGI, 2015).

La relevancia que reviste este tema, consiste en que el fracaso de un franquiciante podría afectar por el cierre de una sola empresa. Aunque el efecto dominó recae en los franquiciatarios dado que múltiples inversionistas obtuvieron préstamos para adquirir franquicias (Jalife, 2016) e invirtieron en lo que creían era un negocio probado y solvente (Buchan et al., 2015), estable y duradero y que brindaría apoyo, capacitación y asistencia técnica (Weaven et al., 2018).

Sin embargo el daño es mayor cuando son unidades franquiciadas que no fueron recompradas, ya que implica daños catastróficos y potencialmente severos (Buchan et al., 2015); destruyendo así el sustento económico, comercial y familiar del franquiciatario, afectando en consecuencia a los empleados y a toda la cadena de suministro (Emerson, 2015). Por ello el desconocimiento del fracaso del franquiciante ha limitado las investigaciones empíricas y la evolución de normas o políticas que aborden cualquier deficiencia de la ley (Buchan, 2013), obstaculizando asíla regulación del sector; por consecuencia, se abren espacios a empresas franquiciantes fraudulentas.

Se destaca también la insuficiente información científica y de divulgación en este tema en México; esto en contraste con el constante pronunciamiento sobre la bonanza, beneficios y ventajas en la literatura del sector franquicias; es así como se plantea la pregunta de esta investigación: ¿cuál es la tasa de mortalidad de la MiPyME franquiciante mexicana del sector comercio y servicios durante el período 2005-2014?

Por lo anterior, el propósito de esta investigación consiste en determinar la tasa real del fracaso de las MiPyME que se ofertaron como franquiciantes durante el período 2005-2014. Para ello se realizó una investigación exploratoria y documental de fuentes secundarias sobre este tema, considerando una muestra de 7037 Mipymes franquiciantes mexicanas del sector comercio y servicios.

El presente estudio es pionero en México y adquiere relevancia debido a que el fracaso del franquiciante ha sido escasamente abordado tanto en investigaciones nacionales como internacionales, pues ha sido minimizado por los defensores del sector al promover este formato de negocios como una atractiva fórmula comercial que disminuye los riesgos del inversionista-franquiciatario y que incrementa la expansión y supervivencia de una MiPyME con potencial de crecimiento.

Este trabajo se divide en cuatro apartados: en el primer apartado se desarrolla la revisión de literatura en cuanto a la definición del modelo de franquicias, el fracaso empresarial, los principales 
problemas en la investigación del fracaso, el fracaso del franquiciante y las teorías utilizadas, el fracaso del franquiciante en México, así como la evolución del sector franquicias en México; en el segundo se explica cómo se determinó la muestra, la recolección de datos y el método de análisis; en el tercero se presentan y analizan los resultados y se finaliza con la respectiva discusión y conclusiones de esta investigación.

\section{Revisión de literatura}

\section{El modelo de franquicias}

La definición de franquicia es amplia e indefinida dado que no existe una conceptualización única (Altinay and Miles, 2006) por la diversidad de enfoques como son el doctrinal (económico-comercial y jurídico), normativo o institucional (Bermúdez, 2001) e incluso por país (Dant, Perrigot and Cliquet, 2008). En México, la Asociación Mexicana de Franquicias -AMF- (2018) la define como:

\begin{abstract}
formato de negocio dirigido a la comercialización de bienesy servicios, según el cual, una persona física o moral (franquiciante), concede a otra (franquiciatario), por un tiempo determinado, el derecho de uso de una marca, trasmitiéndole asimismo los conocimientos técnicos necesarios que le permitan comercializar determinados bienes y servicios con métodos comerciales y administrativos uniformes, a cambio de determinadas contraprestaciones económicas.
\end{abstract}

Por otra parte Mishra (2017) la define como un modelo de negocio que proporciona una ventaja sostenida y competitiva para el franquiciante; no obstante, también representa desventajas para ella misma (López y Ventura, 2001). Ketchen, Short y Combs (2011) definen al franquiciante como el emprendedor exitoso o empresario con experiencia que construye el modelo de negocios a franquiciar, quien define la estrategia y gestiona la red de unidades franquiciadas, concediendo al franquiciatario el derecho de usar por un período determinado la marca y los demás elementos de propiedad intelectual; es el que recluta posibles franquiciatarios con o sin experiencia comercial previa, ya que confía en la capacidad de su modelo comercial para compensar cierta inexperiencia (Weaven et al., 2018) puesto que depende de la unidad franquiciada para maximizar sus ingresos en una región geográfica específica (Grewal et al., 2011). Aunque "la franquicia a menudo se promueve como una alternativa menos arriesgada a las pequeñas empresas independientes" (Buchan and Frazer, 2013, p. 16), Holmbergy Morgan (2003) coincidieron que la realidad demuestra que las cadenas no siempre son exitosas y se pueden observar altas tasas de fallas; aun con puntos de venta propios (Buchan et al., 2015).

Esas fallas en nuevos franquiciantes representan un porcentaje significativo (Holmberg and Morgan, 2003) dado que dejan de franquiciar en los primeros tres años (Mariz-Pérez, 2012) y esto aunado a que los limitados artículos sobre el fracaso de los franquiciantes no consideran a los franquiciatarios afectados (Eljelly and Mansour, 2001), distorsionando así los resultados del sector.

\section{El fracaso empresarial}

Ooeghe y De Prijcker (2008) presentan la inexistencia de un concepto uniforme del fracaso debido a las diferentes aristas y aproximaciones conceptuales. Así pues, Hill, Perry y Andes (1996) lo definieron como insolvencia técnica o falta de liquidez. En cuanto a Mures, García y Vallejo (2012) clasificaron el fracaso en económico (baja rentabilidad), financiero (problemas de liquidez o insolvencia técnica) y jurídico (sentido de quiebra); a su vez Romero (2013) identificó diversas posturas como la insolvencia, la suspensión de pagos o el fracaso financiero hasta la quiebra. Por otra parte Ooeghe y De Prijcker (2008) señalan que el fracaso se presenta por deficiencias de planeación, gestión y distintos patrones de crecimiento en las empresas como son: (i) aquellas que lo hacen de forma acelerada y continua (gacelas); (ii) las que demoran hasta que despegan fuerte; (iii) las que crecen moderadamente, pero en forma sostenida; (iv) las que crecen rápido, pero de forma inestable y (v) las que crecen a través de un portafolio de emprendimientos del emprendedor.

En el contexto de la franquicia Rodríguez-Rad y Navarro-García (2007) afirman que el fracaso del franquiciante es cualquier hecho que suponga que la marca abandona el modelo de franquicia ya 
sea porque desaparece la empresa del mercado o porque decide dejar de franquiciar; en contraparte, Macías (2015) lo asume como la ausencia en el registro de la asociación por compra o adquisición de otra empresa y por liquidación.

\section{Principales problemas en la investigación del fracaso del franquiciante}

La investigación exploratoria documental agrupó los seis principales problemas en la investigación del fracaso en el franquiciante:

(i) por deficiencias en la información del franquiciante debido a la falta de transparencia de las fuentes sobre tasas del fracaso de franquiciatarios (Hoy, 1994); pues las fallas de los franquiciatarios pueden ser enmascaradas al encubrir las cifras, los motivos o por la distorsión en la definición de la falla-fracaso, así como la recolección de los datos por parte de los responsables de los anuarios y directorios del sector (Dant, Grünhagen and Windsperger, 2011).

(ii) Por deficiencias en la consistencia y veracidad en la información en cuanto a estadísticas no corroboradas (Stanworth, Purdy and Price, 1997), por datos potencialmente incompletos e inexactos presentados por los franquiciantes (Cross, 1998) y por literatura fragmentada (Stanworth, Purdy and Price, 1997), lo que ocasiona que los investigadores utilicen bases de datos de anuarios en lugar de datos suministrados por el gobierno (Stanworth and Purdy, 1992).

(iii) La escasa investigación por país en la que el liderazgo de Estados Unidos (Dant, Perrigot and Cliquet, 2008) ha sido constante, incluso en la International Society of Franchising - ISoF(Young and McIntyre, 2011). Sin embargo la investigación sobre el fracaso en las franquicias es escasa especialmente en Latinoamérica dado que aparte de Reino Unido, Canadá, Francia, Australia, España y Brasil, los estudios del fracaso se limitaron solo a Estados Unidos (Buchan et al., 2015; Macías, 2015).

(iv) Por limitaciones geográficas debido a las jurisdicciones, el marco legal y regulatorio de cada país, la madurez del sector, el apoyo de las instituciones y del gobierno, los factores culturales, el nivel de competitividad (World Bank, 2015), la competencia gerencial del franquiciante (McCartanQuinn and Carson, 2003) entre otros, siendo necesaria una investigación intercultural que extrapole resultados más allá de la comparación con Estados Unidos para crear coherencia conceptual, empírica y metodológica (Façanha et al., 2013).

(v) Las investigaciones se han abordado desde diferentes disciplinas tales como la estrategia, el marketing, el emprendimiento y la economía (Beere, 2017), siendo necesario incrementar las relacionadas al fracaso puesto que "identificar los problemas, facilita establecer parámetros y restricciones que condicionen un diseño de investigación para futuras investigaciones empíricas" (Buchan et al., 2015, p. 315).

(vi) Por el ciclo de vida del franquiciante dado que el fracaso es el resultado de una serie de acciones y decisiones que llevaron un determinado tiempo; de ahí que es necesario identificar la fase en la que ocurrió la falla (Buchan et al., 2015); sin embargo el ciclo de vida varía por autor, país y año e incluso por reputación de la marca y del franquiciante (Cortes-Castillo, 2019).

\section{El fracaso del franquiciante}

El fracaso del franquiciante presenta escasa atención académica (Macías, 2015), científica, profesional y gubernamental (Buchan, 2013) sobre todo de la naciente MiPyME franquiciante (Bordonaba, Palacios and Polo, 2009). Estudios realizados en Australia, Estados Unidos, Reino Unido y Francia coincidieron en que el fracaso del franquiciante es continuo y con un gran alcance (internacional) que afecta fuertemente al sector (Buchan etal.,2015); de ahí que es necesario realizar investigaciones en otros mercados distintos al de Estados Unidos (Dant, Perrigot and Cliquet, 2008).

El modelo de franquicias no es lo que se prometió puesto que muchas empresas fracasaron a pesar de franquiciar; otras abandonaron el modelo, después de intentarlo durante algunos años (Lafontaine and Shaw, 1998); pese a ser una estrategia atractiva de crecimiento para la MiPyME genera importantes problemas (Kirby and Watson, 1999), pues la tasa de mortalidad de franquiciantes y franquiciatarios son comparables a las tasas de fracaso en otros negocios (Blair and Lafontaine, 2005); esto es un desafío para el franquiciante 
debido a la creciente falta de recursos adecuados, las condiciones ambientales, las reglas, rutinas y estructuras que reducen la eficiencia y la eficacia de la gestión (Dobbs et al., 2012).

Buchan y Frazer (2013) identificaron diferentes estudios del fracaso tanto del franquiciante como del franquiciatario por tema y país: de 66 estudios, 17 abordaron la falla del franquiciatario y 31 se enfocaron en las fallas del franquiciante; en donde sobresalen las disciplinas del derechoy la economía. En años posteriores se logró identificar 11 estudios adicionales en derecho, economía, administración, marketing, contabilidad y finanzas, de los cuales solo 2 abarcaron múltiples disciplinas.

\section{Teorías sobre el fracaso de las franquicias}

Diversas teorías se han utilizado en los estudios del fracaso. La teoría de la agencia y la escasez de recursos facilitó determinar cómo los franquiciantes afectaron el fracaso del franquiciatario (Michael and Combs, 2008), también para identificar que la ausencia de estructura del franquiciante aumentaría la probabilidad de falla (Madanoglu and Castrogiovanni, 2018).

Por su parte con la teoría de la agencia y la escasez de recursos y con la teoría de los costos de transacción se logró identificar el perfil del franquiciante más proclive al fracaso (Macías, 2015). Las teorías de la nueva economía institucional, de los recursos y capacidades y de las señales facilitaron un modelo econométrico multivariado para la determinación del fracaso del modelo de franquicias en México (Cortes-Castillo, 2019). Con la teoría contractual se descubrió que los contratos no se ajustaban a las circunstancias particulares de los agentes y que, además, estos no varían con el tiempo (Lafontaine and Bhattacharyya, 1995).
La teoría de Bull y Willard reveló la existencia de riesgos y ventajas inherentes en las franquicias (Hoy, 1994); la teoría de la gestión contemporánea demostró que entre las principales causas de fracaso esta la competencia gerencial del dueño del negocio (Beaver and Jennings, 2005). En contraparte, la teoría de la ecología organizacional de Hannan y Freeman (1977) facilitó la comprensión de cómo las fuerzas dan forma a las estructuras de las empresas a lo largo del tiempo; finalmente Calderón-Monge y Pastor-Sanz (2017) se apoyaron en la teoría de la sustentabilidad corporativa dado que promueve la protección del medio ambiente y la equidad social, logrando el desarrollo económico cuando se implementa un modelo de gestión que fomente el diálogo y forje relaciones con las partes interesadas.

\section{Evolución del sector franquicias en México}

México forma parte de los principales destinos para la expansión de franquiciantes extranjeros al triplicar su actividad en Latinoamérica por su estabilidad y crecimiento sostenido (AMF, 2018). Los franquiciantes son parte activa del modelo de franquicias al generar la mayor parte de ingresos de la AMF debido a la afiliación y renovación a una asociación.

El crecimiento de los franquiciantes en México inicia en 1984 con cuatro empresas y el nacimiento de la AMF en 1989, incrementando a 150 marcas entre 1992 a 1994 (de las cuales el 40 \% eran nacionales) (Feher, 2015); porcentaje que ascendió al $60 \%$ para el 2000 y manteniéndose en niveles del $85 \%$ a partir de 2007 (May, Aguileray Loy, 2011). En 2007 se incrementó el $21 \%$, año en el que se anuncia el Programa Nacional de Franquicias - PNF-; siguiendo con tasas del $15 \%$ en 2008 y el $47 \%$ en 2009; sin embargo se registra una contracción del $17 \%$ en 2011 y una continua disminución en el siguiente sexenio. 
Tabla 1. Evolución de las empresas franquiciantes en México

\begin{tabular}{lll}
\hline Año & Número de empresas franquiciantes & Porcentaje de incremento contra el año pasado \\
\hline 2005 & 450 & $38 \%$ \\
\hline 2006 & 619 & $21 \%$ \\
\hline 2007 & 750 & $15 \%$ \\
\hline 2008 & 860 & $47 \%$ \\
\hline 2009 & 1262 & $23 \%$ \\
\hline 2010 & 1550 & $-17 \%$ \\
\hline 2011 & 1280 & $7 \%$ \\
\hline 2012 & 1370 & $-0,4 \%$ \\
\hline 2013 & 1365 & $6 \%$ \\
\hline 2014 & 1450 & $-24 \%$ \\
\hline 2015 & 1096 & $-4 \%$ \\
\hline 2016 & 1050 & $24 \%$ \\
\hline 2017 & 1300 & $24 \%$ \\
\hline
\end{tabular}

Nota: incluye el total de empresas franquiciantes (nacionales y extranjeras).

Fuente: elaboración propia por parte de los autores.

No obstante, esta información fue elaborada con datos explorados ante la ausencia de un organismo que facilite información consistente y confiable; como es el caso de Australia, donde la información es pública a través del Consejo de Franquicias y presentada por la Griffith University de Queensland (Frazer et al., 2016).

\section{El fracaso del franquiciante en México}

Durante el período 2000 a 2013 las franquicias en México han sido escasamente estudiadas. Alba, Macho y Zúñiga (2015) identificaron entre 1377 tesis y 323 libros que abordaron el tema en un 0,37 \%; también Cavazos y Ayup (2014) exponen que la participación de la franquicia en el sector de la actividad económica aún no se precisa; mientras que Guerrero, Armenteros y Medina (2014) observaron vacíos en la información en cuanto a los factores críticos de éxito, el desarrollo a nivel territorial (estatal), el incremento en ventas, empleo y utilidades de las franquicias mexicanas.

Por su parte Cortes-Castillo (2019) identificó durante el período 2002 a 2017 alrededor de 73 estudios sobre empresas franquiciantes tras una revisión exhaustiva de los estudios empíricos realizados en México (tesis, artículos y ponencias), destacando una marcada ausencia de estudios de Mipymes franquiciantes fallidas. Entre los hallazgos más relevantes hay que mencionar que Ayup y Cavazos (2015) observaron que mientras la comercialización de franquicias aumenta, el total de estos establecimientos creció de manera inversa; esto aunado a que ninguna dependencia tiene datos fáciles de consultar y que puedan ser usados en futuras investigaciones (Alvídrez y Reyes, 2010), poniendo en duda la veracidad de la información vertida por asociaciones y expertos dado que ninguna investigación ha logrado confirmar las cifras y los datos emanados por estos (May, Aguilera y Loy, 2011).

Con base a lo anterior, en México, el avance de las investigaciones de carácter científico de las franquicias ha sido lento (Guerrero, Armenteros y Medina, 2014). Esto ha imposibilitado la investigación, así como el avance paralelo en la evolución del sector (Batres, 2009), confirmando vacíos en la información sobre el fracaso del 
franquiciante mexicano y las áreas de oportunidad durante la gestión de la red; pues se confunde la tasa de crecimiento y éxito con el número de franquiciantes nuevos que ingresan cada año, ya que la tasa neta está muy por debajo de las publicadas por las asociaciones de franquicias al no considerar a los franquiciantes que fracasan y abandonan el modelo (Lafontaine and Shaw, 1998).

Así, Cortes-Castillo (2019) exploró fuentes terciarias respecto a la evolución del fracaso o abandono del franquiciante en México durante el período 2007 a 2018 en el que confirmó una serie de evidencias que señalan de forma latente dicho fracaso. Por su parte Guerrero, Armenteros y Medina (2014) identificaron en una muestra de 52 franquiciantes de la Comarca Lagunera a 8 que habían abandonado el modelo de franquicias, 5 ya no existían, de las restantes 7 no habían logrado vender nada o no estaban preparadas y la mayoría no pertenecían a la AMF. Entretanto AyupyCalderónMonge (2014) concluyeron que los franquiciantes incrementan el número de unidades, pero no logran el crecimiento sostenido tras explorar una muestra de 911 franquiciantes durante el período 2002 a 2008.

Por otra parte la AMF pronosticó que 25 o 26 franquiciantes dejan de operar al año, al calcular una tasa del $2 \%$ de fracaso ${ }^{1}$. Aunque es una tasa muy baja, si se compara contra el porcentaje de contracción en el crecimiento de franquiciantes mostrado en la tabla $1(-17 \%,-24 \%$ y $-4 \%)$; luego de identificar la tasa de fracaso de otros países entre ellos España cuya tasa osciló en promedio entre el 14 y el 35 \% (Mariz-Pérez, 2012), se estableció para México el supuesto de una tasa promedio de fracaso de franquiciantes menor al $10 \%$ debido a los apoyos que el gobierno federal brindó al sector franquicias y que no se dieron de forma similar en ningún otro país. La inferencia ante la ausencia de investigaciones científicas que determinen la tasa de fracaso de franquiciantes en México permitió plantear la siguiente hipótesis:

$\mathrm{Hi}_{1}$ : la tasa promedio de fracaso de la MiPyME franquiciante mexicana durante el período 2005 2014 fue menor al $10 \%$.

Siendo la hipótesis nula:
$\mathrm{Ho}_{1}$ : la tasa promedio de fracaso de la MiPyME franquiciante mexicana durante el período 2005 2014 fue igual al $10 \%$.

Por consiguiente, se plantea la hipótesis 2:

$\mathrm{Hi}_{2}$ : el total de las empresas que adoptaron el modelo de franquicias en México durante el período 2005-2014 es menor a las empresas que se encontraban vigentes en 2014.

Siendo la hipótesis nula:

$\mathrm{Ho}_{2}$ : el total de empresas que adoptaron el modelo de franquicias en México durante el período 2005-2014 es igual a las empresas que se encontraban vigentes en 2014.

\section{Metodología}

Para fines de la presente investigación el fracaso del franquiciante se asumió como franquiciante fallido no sobreviviente (Frazer, 2001) al dejar de aparecer en los anuarios de Entrepreneur (Macías, 2015); registrando como el año (tiempo) de salida el año anterior al último año en que el franquiciante figuraba en el anuario (Dobbs etal., 2012) debido al paso del tiempo entre el momento de la recolección de datos y la publicación del directorio.

Con el fin de determinar la tasa real de mortalidad de Mipymes franquiciantes mexicanas durante el período 2005-2014 se realizó la investigación exploratoria descriptiva bajo un diseño de investigación no experimental longitudinal con 10 cortes (2005-2014), ex post facto evolutivo, explorando el fracaso después de que había sucedido para poder identificar el número de empresas franquiciantes. La técnica de recolección de datos fue el análisis de datos secundarios ante la ausencia de una institución que brindará información respecto a la mortalidad del sector. Por tanto, se procedió con la consulta del directorio de Entrepreneur durante el período 2006-2016; información utilizada previamente en investigaciones científicas internacionales

\footnotetext{
${ }^{1}$ En palabras de la AMF debido a los problemas entre la comunicación del franquiciante y el franquiciatario.
} 
(Kosová and Lafontaine, 2010; Kacker and Perrigot, 2016), las cuales integran características y datos secundarios de los franquiciantes brindados en forma voluntaria.

El diseño muestral consideró el censo de las empresas franquiciantes mexicanas bajo los siguientes criterios: dentro del ciclo de vida (nacientes, jóvenes y maduras) del sector comercio y servicios afiliadas y no afiliadas a una asociación mexicana de franquicias pertenecientes o no a un programa de gobierno relacionado al sector franquicias; además de franquicias maestras con oficinas en México en el año de publicación, excluyendo a franquiciantes extranjeros con oficinas por fuera del país; en total se analizó la evolución de 7037 empresas franquiciantes por un período de 10 años.

En la mortalidad de las Mipymes franquiciantes se identificaron: primero las empresas sobrevivientes al comparar la edición del año anterior contra el siguiente año en que el franquiciante figuraba en el anuario, procedimiento sugerido por Dobbs et al. (2012) debido al paso del tiempo entre el momento de la recolección de datos y la publicación del directorio; luego se marcaron las empresas que dejaron de aparecer en el año más reciente, sin embargo se detectó la existencia de empresas que abandonaron el modelo de franquicias y posteriormente volvieron a aparecer en las publicaciones de los años siguientes; por ello se procedió a validar a través de una investigación exhaustiva el rastreo por medios digitales (posicionamiento Web, redes sociales) y llamadas telefónicas la permanencia de dichas empresas.

Con esta pesquisa se identificaron tres tipos de empresas franquiciantes: (i) empresas franquiciantes que abandonan el modelo de franquicias y siguen operando sin franquiciar; (ii) empresas franquiciantes sobrevivientes a las cuales se integran aquellas que abandonaron el modelo de franquicias por un tiempo y regresaron al sector y (iii) empresas que dejaron de operar (fracasaron).

\section{Resultados}

Los resultados del seguimiento de la evolución de las empresas franquiciantes se muestran en la tabla 2 en la que se observa que durante el período 2005-2014 las empresas franquiciantes crecieron en promedio un $37 \%$; destacando un impulso estratosférico en 2006 (240\%), 2007 (30\%) y 2010 (53 \%) durante la vigencia del PNF; sin embargo también se denota una caída vertiginosa del -54\% en 2009, lo cual podría deberse al impacto de la crisis económica estadounidense y su impacto en la economía mexicana; al igual que del -4 \% en 2012 , que también coincide con una crisis económica a la que se le denominó la crisis del euro y que impactó a las economías emergentes como México.

Además el seguimiento de la evolución permitió identificar que fueron 801 las empresas que dejaron de operar y cuya proporción del total de empresas que iniciaron $(801 / 7037)$ dio como resultado el $11,38 \%$. Por ello se dispone de evidencia para rechazar la hipótesis nula $\mathrm{Ho}_{1}$ : $\mu=10 \%$, así como la hipótesis de investigación $\mathrm{Ho}_{1}$ : $\mu<10 \%$, concluyendo que la tasa de mortalidad de las Mipymes franquiciantes mexicanas fue mayor al $10 \%$.

Esto superó el pronóstico de la $\mathrm{AMF}$ (Mundoadmi, 2013) al calcular el fracaso entre 25 o 26 franquicias en el país ( $2 \%$ ), cuando en realidad la cifra fue superior a las 80 empresas por año; sin contar aquellas empresas franquiciantes mexicanas y empresas franquiciantes extranjeras que dejaron de operar y que no se registraron en el anuario, lo que representa un $310 \%$ superior a las cifras establecidas por la AMF. 
Tabla 2. Análisis del censo de empresas franquiciantes mexicanas durante el período 2005-2014

\begin{tabular}{|c|c|c|c|c|c|c|}
\hline Año & $\begin{array}{c}\text { Total de } \\
\text { empresas } \\
\text { franquiciantes } \\
\text { mexicanas }\end{array}$ & $\begin{array}{l}\text { Empresas que } \\
\text { abandonaron } \\
\text { el modelo de } \\
\text { franquicias y } \\
\text { continuaron } \\
\text { operando* }\end{array}$ & $\begin{array}{c}\text { Empresas } \\
\text { que dejaron } \\
\text { de operar } \\
\text { (mortalidad)* }\end{array}$ & $\begin{array}{c}\text { Empresas } \\
\text { franquiciantes } \\
\text { sobrevivientes* }\end{array}$ & $\begin{array}{l}\text { Crecimiento } \\
\text { absoluto real } \\
\text { de empresas } \\
\text { franquiciantes } \\
\text { mexicanas }\end{array}$ & $\begin{array}{l}\text { Crecimiento } \\
\text { relativo real } \\
\text { de empresas } \\
\text { franquiciantes } \\
\text { mexicanas** }\end{array}$ \\
\hline 2005 & 370 & 85 & 51 & 234 & 98 & \\
\hline 2006 & 373 & 7 & 13 & 353 & 333 & $240 \%$ \\
\hline 2007 & 612 & 37 & 52 & 523 & 434 & $30 \%$ \\
\hline 2008 & 807 & 67 & 100 & 640 & 473 & $9 \%$ \\
\hline 2009 & 862 & 177 & 146 & 539 & 216 & $-54 \%$ \\
\hline 2010 & 711 & 104 & 86 & 521 & 331 & $53 \%$ \\
\hline 2011 & 698 & 97 & 61 & 540 & 382 & $15 \%$ \\
\hline 2012 & 867 & 137 & 114 & 616 & 365 & $-4 \%$ \\
\hline 2013 & 802 & 104 & 72 & 626 & 450 & $23 \%$ \\
\hline 2014 & 935 & 81 & 106 & 748 & 561 & $25 \%$ \\
\hline Total & 7037 & 896 & 801 & 5340 & 364 & \\
\hline Porcentaje & $100 \%$ & $13 \%$ & $11 \%$ & $76 \%$ & & $37 \%$ \\
\hline
\end{tabular}

Nota: *no se integran las franquicias maestras extranjeras en México ni aquellas en propiedad de empresarios mexicanos; ${ }^{\star \star}$ el total es la suma promedio.

Fuente: elaboración propia por parte de los autores.

Tabla 3. Estadístico univariante prueba t para muestras relacionadas

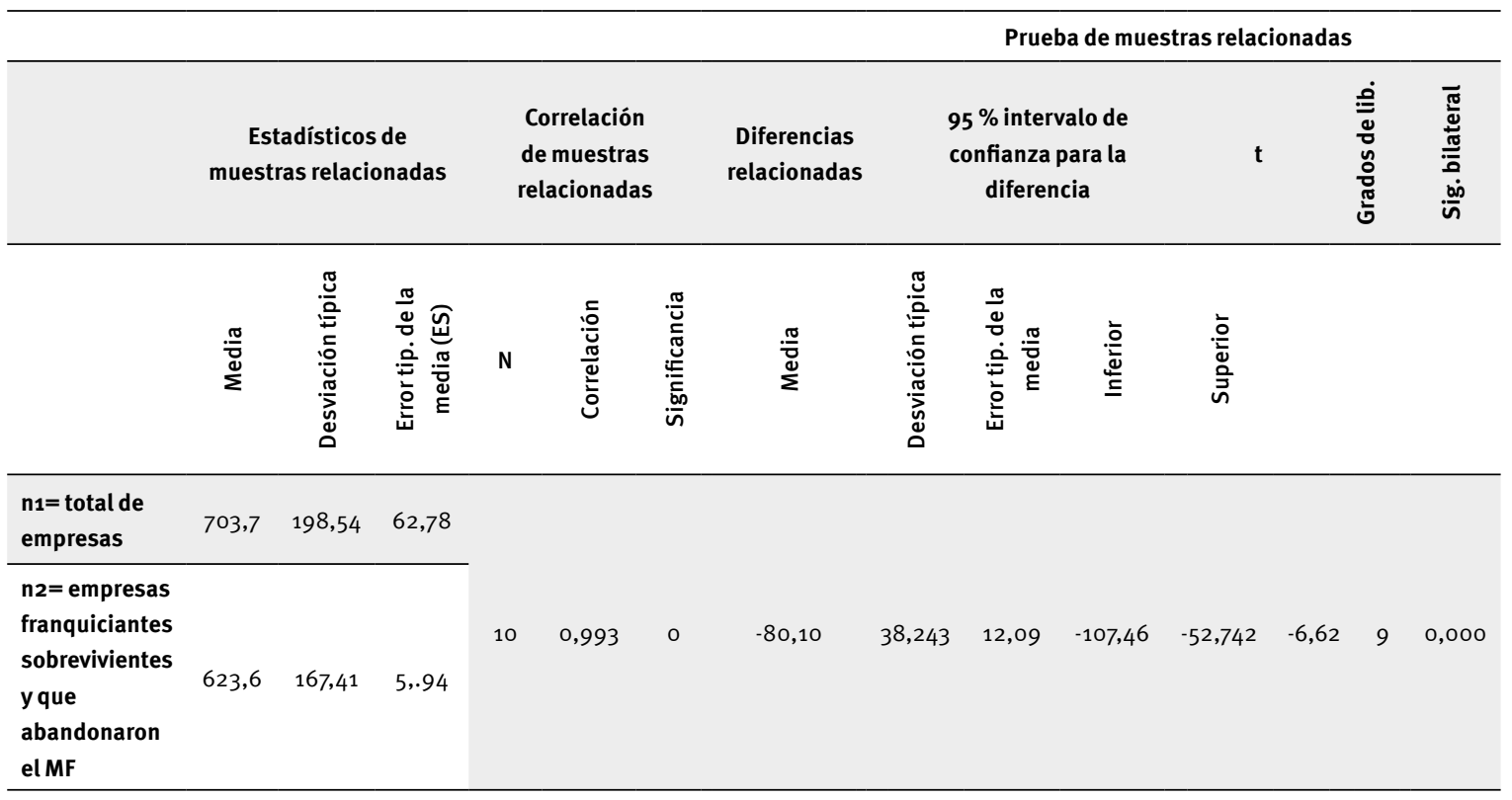

Nota: *se trabajó con el total de empresas sobrevivientes y que abandonaron, así como con el total de empresas del período de estudio.

Fuente: elaboración propia por parte de los autores. 
En la tabla 3 los estadísticos descriptivos indican que el grupo inicial de empresas franquiciantes experimentaron una disminución significativa $\left(\mathrm{M}=703,7, \mathrm{ES}^{2}=62,785\right)$ después de un período de 10 años $(M=623,6, E S=52,94, t(9)=-6,623, p<0,05)$, presentando una estimación de fracaso media de 80,1 de empresas franquiciantes por año.

La prueba estadística de la prueba t establece el cumplimiento al supuesto de distribución normal, mismo que se comprueba con la prueba de normalidad de Shapiro-Wilk (muestras menores de 50 y mide la fuerza de ajuste de una recta) con los siguientes pasos:

1. Formulación de hipótesis e hipótesis nula:

$\mathrm{Hi}=\mathrm{la}$ distribución de las medias de ambos grupos no siguen una distribución normal:

\Нo: $\mu \rrbracket \_D \neq 0$

Ho=la distribución de las medias de ambos grupos siguen una distribución normal:
『Нo: $\mu \rrbracket \_D \sim N$

2. Nivel de significancia $=5 \%=0,05$.

3. Elección de prueba estadística: prueba de normalidad de Shapiro-Wilk.

4. Estimación de p-valor.

5. Toma de decisión:

criterio opción:

Si $\mathrm{p}<0,05$ se debe rechazar Ho (hipótesis nula), por lo tanto se considera la hipótesis alternativa de la ausencia de distribución normal.

Si p>0,05 se debe aceptar Ho (hipótesis nula).

El resultado de la prueba estadística de ShapiroWilk establece un nivel de significancia de $5 \%$, el cual se dispone de evidencia para aceptar la hipótesis nula que la distribución de las variables analizadas es igual a la distribución normal puesto que el valor $p$ de las muestras es mayor a 0,05 (tabla 4).

Tabla 4. Prueba de Shapiro-Wilk para dos muestras

\begin{tabular}{llll}
\hline \multicolumn{1}{c}{ Concepto } & $\begin{array}{c}\text { Total de empresas } \\
\text { franquiciantes }\end{array}$ & $\begin{array}{c}\text { Total de empresas } \\
\text { sobrevivientes franquiciantes } \\
\text { y que abandonaron la AMF }\end{array}$ & $\begin{array}{c}\text { Empresas franquiciantes } \\
\text { que dejaron de operar }\end{array}$ \\
\hline $\mathrm{N}$ (años) & 10 & 10 & 10 \\
\hline Media & 623,6 & 703,7 & 80,1 \\
\hline Desviación estándar & 167,412 & 198,542 & 38,243 \\
\hline Varianza & 28026,711 & 39419,122 & 1462,544 \\
\hline Z de Kolmogórov -Smirnov & 0,643 & 0,600 & 0,390 \\
\hline P valor (sig. asintót. (bilateral)) & 0,803 & 0,864 & 0,998 \\
\hline Shapiro-Wilk & 0,875 & 0,884 & 0,997 \\
\hline P valor (sig.) & 0,115 & 0,146 & 0,994 \\
\hline a. La distribución de contraste es la normal. & & \\
\hline b. Se han calculado a partir de los datos. & & & \\
\hline
\end{tabular}

Fuente: elaboración propia por parte de los autores.

${ }^{2}$ ES: error estándar. 
En consecuencia, la prueba de diferencia de medias con la que se realizó la comparación entre el número total de empresas que eran franquiciantes en $2014\left(n_{1}\right)$ con las empresas sobrevivientes y las que abandonaron temporalmente el modelo pero que regresaron en los años siguientes y se encontraban vigentes en $2014\left(\mathrm{n}_{2}\right)$ mostró como resultado un valor $p=0,000$ con un nivel de significancia del $5 \%(-6,623$, sig. bilateral 0,000 $<0,05$ ); por ello se dispone de evidencia para rechazar la hipótesis nula $\mathrm{Ho}_{2}: \mu n_{1}=\mu n_{2}$, concluyendo que el total de empresas que adoptaron el modelo de franquicias en México durante el período 2005-2014 es diferente a las empresas que se encontraban vigentes en 2014; esto se mostró en el análisis descriptivo de la evolución de las empresas franquiciantes en la tabla 2.

\section{Discusión}

Es importante destacar que el porcentaje del $11 \%$ de franquiciantes que dejaron de operar en México (tabla 2) es bajo en comparación al de los Estados Unidos en 1998, al identificar una tasa del $70 \%$ durante el período de 12 años (Lafontaine and Shaw, 1998) y del $80 \%$ durante el período de 1985 a 2005 (Dobbs et al., 2012); de Australia donde Connors (2010) identificó un promedio de 6 franquiciantes fallidos por cada franquiciante exitoso; en Canadá, de modo similar, Kosová y Lafontaine (2010) observaron una tasa de alrededor del $60 \%$ durante un período de 10 años; en Francia, les correspondió a Perrigot, Cliquet y Mesbah (2004) comprobar un fracaso del 57 \% y en Reino Unido el 50 \% (Lafontaine and Shaw, 1998); de ahí que solo pueda considerarse similar al de España, respecto a una tasa del 9,5 \% (Calderón-Monge et al., 2017) para un período de 10 años. Aunque por las limitaciones en la recolección de datos es probable que el resultado de esta investigación se deba considerar únicamente como un acercamiento a la realidad dado que no todas las Mipymes franquiciantes realizaron su registro en la AMF debido a que esto es voluntario.

\section{Conclusiones}

Los resultados obtenidos cumplieron con los objetivos planteados y mostraron evidencia empírica de que la tasa de fracaso de la MiPyME franquiciante mexicana durante el período 2005-2014 fue superior al $10 \%$; corroborando que como consecuencia de la complejidad en el acceso a la información de las empresas franquiciantes, el desconocimiento del estatus de los franquiciatarios (nuevos, actuales y que dejaron de operar) y al ocultamiento o manipulación de los datos expresados por los franquiciantes durante un período de 10 años se identificó un $11 \%$ de Mipymes (801) que dejaron de operar y un $13 \%$ de Mipymes (896) que abandonaron el modelo de franquicias; además no se cuenta con estimaciones reales de la evolución de las empresas de este sector, poniendo en tela de juicio el considerar al modelo de franquicias como una alternativa menos arriesgada para las pequeñas empresas independientes (franquiciatarios e inversionistas) y como una opción de rápida expansión y supervivencia para la MiPyME franquiciante; aquí se cubre parte de un vacío en el conocimiento de esta estrategia de expansión ante la escasez de investigaciones científicas que aborden dicho tema en México.

Cabe mencionar las limitantes identificadas en las bases de datos utilizadas: (i) la ausencia de un organismo que brinde información veraz del número de franquiciantes fallidos durante el ciclo de vida; (ii) los franquiciantes que cambiaron el nombre de la marca y razón social y continuaron operando y (iii) un posible escenario de información oculta o sesgada del franquiciante por la falta de regulación del sector; al igual que la ausencia de categorías específicas en las bases de datos; por ello el análisis fue univariante puesto que se determina la estimación de la tasa promedio de franquiciantes, explorando como se manifiesta una variable en una constante.

Finalmente entre las futuras líneas de investigación se encuentran la determinación del impacto del fracaso del franquiciante, la caracterización del franquiciante que dejó de operar y los que abandonaron el modelo de franquicias y los factores asociados al fracaso del franquiciante mexicano. 


\section{Referencias}

Alba, M., Macho, M.y Zuñiga, G. (2015). Publicaciones sobre la Mipyme: tesis y libros en México. Ciudad de México, México: Publicaciones Empresariales UNAM.

Altinay, L. and Miles, S. (2006). International franchising decision-making: An application of stakeholder theory. The Service Industries Journal, 26(4), 421-436.

Alvídrez, R. y Reyes, G. (2010). Franquicia, oportunidad de crecimiento en pymes del giro restaurantero de la ciudad de Chihuahua. En XIV Congreso Internacional de Investigación en Ciencias Administrativas, Monterrey, Nuevo León, México.

AMF. (2018). Esta es la fórmula del éxito de una franquicia. Recuperado de http:// franquiciasdemexico.org.mx/formula-exitode-una-franquicia/.

Ayup, J. y Calderon-Monge, E. (2014). Señales de valor de marca de las franquicias en México. $\mathrm{Su}$ efecto en el crecimiento del sistema franquiciador. Estudios Gerenciales, 30(131), 134-144.

Ayup, J. y Cavazos, A. (2015). La franquicia en Iberoamérica. Estado y tendencias. Ciudad de México, México: Plaza y Valdés.

Batres, R. (2009). Un modelo para la valuación de intangibles. Aplicación a las franquicias de la industria restaurantera en México. Valencia, España: Universitat Politècnica de València.

Beaver, G. and Jennings, P. (2005). Competitive advantage and entrepreneurial power. The dark side of entrepreneurship. Journal of Small Business and Enterprise Development, 12(1), 9-23.

Beere, R. (2017). The role of franchising on industry evolution: assessing the emergence offranchising and its impact on structural change. Dublin, Ireland: Palgrave Macmillan.
Bermúdez, G. (2001). Satisfacción y fidelidad en la relación franquiciada franquiciador. Málaga, España: Universidad de Málaga.

Blair, D. and Lafontaine, F. (2005). The economics offranchising. Cambridge, England: Cambridge University Press.

Bordonaba, V., Palacios, L. and Polo, Y. (2009). Franchise firm entry time influence on longterm survival. International Journal of Retail \& Distribution Management, 37(2), 106-125.

Buchan, J. (2013). Franchisees as consumers: Benchmarks, perspectives and consequences. New York, USA: Springer.

Buchan, J. and Frazer, L. (2013). The effect of franchisor failure on franchisees: A review of the literature. En Sixth International Conference on Economics and Management of Networks, Agadir, Morocco.

Buchan, J. et al. (2015). Franchisor Insolvency in Australia: Profiles, Factors, and Impacts.Journal of Marketing Channels, 22(4), 311-332.

Calderon-Monge, E. and Pastor-Sanz, I. (2017). Effects of contract and trust on franchisor performance. Contemporary Economics, 11(4), 383-400.

Cavazos, J.y Ayup, J. (2014). Análisis del inversionista y el emprendedor en el sector franquicias del estado de Tamaulipas. En XIX Congreso Internacional de Contaduría, Administración e Informática, Ciudad de México, México.

Combs, J. et al. (2011). Antecedents and Consequences of Franchising: Past Accomplishments and Future Challenges. Journal of Management, 37(1), 99-126.

Connors, E. (6-7 de febrero de 2010). The Brave New World of Franchising. The Weekend Australian Financial Review, p. 39.

Cortes-Castillo, M.R. (2019). Propuesta de un modelo econométrico multivariado para la determinación del fracaso del modelo de franquicias en México durante el periodo 2005- 
2014 (tesis de posgrado). Universidad Popular Autónoma del Estado de Puebla, Puebla, México.

Cross, J. (1998). Improving the relevance of franchise failure studies. En Stanworth, J. and Purdy, D. (Eds.), Franchising beyond the millennium: Learning lessons from the past (pp. 1-14). Minneapolis, USA: University of St. Thomas.

Dant, R., Perrigot, R. and Cliquet, G. (2008). A cross-cultural comparison of the plural forms in franchise networks: United States, France, and Brazil.Journal Small Business Management, 46(2), 286-311.

Dant, R., Grünhagen, M. and Windsperger, J. (2011). Franchising research frontiers for the twentyfirst century.Journal of Retailing, 87(3), 253-268.

Díez, E. y Rondán, F. (2004). La investigación sobre franquicia. Investigaciones Europeas de Dirección y Economía de la Empresa, 10(3), 71-96.

Dobbs, E. et al. (2012). Time will tell: interaction effects of franchising percentages and age on franchisor mortality rates. International Entrepreneurship and Management Journal, 10(3), 607-621.

Eljelly, A. and Mansour, F. (2001). Predicting private Companies failure in the Sudan. Journal of African Business, 2(2), 23-43.

ENAPROCE. (2018). Encuesta nacional sobre productividad y competitividad de las micro, pequeñas y medianas empresas. Recuperado de https://www.inegi.org.mx/contenidos/ programas / e naproce / 2018 / d oc / ENAPROCE2018Pres.pdf.

Emerson, W. (2016). Franchise Terminations: Good Cause Decoded. Wake Forest Law Review, 51(1), 103-164.

Façanha, O. et al. (2013). Survival of new firms in the Brazilian franchising segment: an empirical study. The Service Industries Journal, 33(11), 1089-1102.
Feher, F. (2015). Historia de las franquicias en México. Entrepreneur. Recuperado de https:// www.entrepreneur.com/article/268970.

Frazer, L. (2001). Why franchisors discontinue franchising but continue operating. International Small Business Journal, 19(3), 29-38.

Frazer, L. et al. (2016). Franchising in Australia 2016. The Franchise Council of Australia. Special Report. Queensland, Australia: Griffith University, Franchise Council of Australia.

Grewal, D. et al. (2011). Franchise partnership and international expansion: a conceptual framework and research propositions. Entrepreneurship Theory and Practice, 35(3), 533-557.

Guerrero, M., Armenteros, M. y Medina, M. (2014). Desarrollo estratégico de las franquicias mexicanas: estudio empírico en la Comarca Lagunera, México. Revista Internacional de Administración y Finanzas, 7(6), 45-61.

Hannan, T. and Freeman, H. (1977). The population ecology of organizations. American Journal of Sociology, 82(5), 929-964.

Hill, T., Perry, E. and Andes, S. (1996). Evaluating Firms in Financial Distress: An Event History Analysis. Journal of Applied Business Research, 13(13), 60-71.

Holmberg, R. and Morgan, B. (2003). Franchise turnover and failure. Journal of Business Venturing, 18(3), 403-418.

Hoy, F. (1994). The dark side of franchising or appreciating flaws in an imperfect world. International Small Business Journal, 12(2), 26-38.

INEGI. (2015). Esperanza de vida de los negocios en México. Recuperado de https://www.inegi.org. $\mathrm{mx} /$ temas/evnm/.

Jalife, M. (7 de diciembre de 2016). 25 años de franquicias en México. El Financiero. 
Jiménez, I. (6 de noviembre de 2015). Las 15 mejores franquicias en México. Forbes México.

Kacker, M. and Perrigot, R. (2016). Retailer use of a professional social media network: Insights from franchising. Journal of Retailing and Consumer Services, 30, 222-233.

Ketchen, D. Jr., Short, J. and Combs, J. (2011). Is franchising entrepreneurship? Yes, no, and maybe so. Entrepreneurship Theory and Practice, 35(3), 583-593.

Kirby, D. and Watson, A. (1999). Franchising as a small business development strategy: a qualitative study of operational and "failed" franchisors in the UK. Journal of Small Business and Enterprise Development, 6(4), 341-349.

Kosová, R. and Lafontaine, F. (2010). Survival and growth in retail and service industries: Evidence from franchised chains. The Journal of Industrial Economics, 58(3), 542-578.

Lafontaine, F. and Bhattacharyya, S. (1995). The role of risk in franchising. Journal of Corporate Finance, 2(1-2), 39-74.

Lafontaine, F. and Shaw, L. (1998). Franchising growth and franchisor entry and exit in the U.S. market: myth and reality. Journal of Business Venturing, 13(2), 95-112.

López, B. y Ventura, J. (2001). Grupos estratégicos en las franquicias españolas: características básicas. Economía Industrial, 340, 163-176.

Macías, J. (2015). Análisis longitudinal del fracaso del franquiciador en el sistema de franquicias español (tesis de posgrado). Universidad de Sevilla, Sevilla, España.

Madanoglu, M. and Castrogiovanni, J. (2018). Franchising proportion and network failure. Small Business Economics, 50(4), 697-715.

Mariz-Pérez, M. (2012). Growth and survival: Evidence from Spanish franchising. ProcediaSocial and Behavioral Sciences, 65(3), 58-63.

May, H., Aguilera, O. y Loy, R. (2011). Aportación e importancia de las franquicias en la economía mexicana: ¿realidad o fantasía? Global Conference on Business and Finance Proceedings, 6(2), 1463-1471.

McCartan-Quinn, D. and Carson, D. (2003). Issues which Impact upon Marketing in the Small Firm. Small Business Economic, 21(2), 201-213.

Michael, C. (2003). First mover advantage through franchising. Journal of Business Venturing, 18(1), 61-80.

Michael, C. and Combs, G. (2008). Entrepreneurial failure: The case of franchisees. Journal of Small Business Management, 46(1), 73-90.

Mishra, C. (2017). Creating and Sustaining Competitive Advantage: Management logics, Business models, and entrepreneurial rent. London, England: Palgrave Macmillan.

Mures, M., García, A. y Vallejo, M. (2012). Análisis del fracaso empresarial por sectores: factores diferenciadores. PECVNIA. Revista de la Facultad de Ciencias Económicas y Empresariales, 53-83.

Ooghe, H. and De Prijcker, S. (2008). Failure processes and causes of company bankruptcy: a typology. Management Decision, 46(2), 223-242.

Perrigot, R., Cliquet, G. and Mesbah, M. (2004). Possible applications of survival analysis in franchising research. The International Review of Retail, Distribution and Consumer Research, 14(1), 129-143.

Rodríguez-Rad, C. y Navarro-García, A. (2007). Una investigación sobre el fracaso de los franquiciadores en España. En Ayala Calvo, J.C. (Ed.), Conocimiento, innovaciónyemprendedores: camino al futuro (pp. 2142-2146). Logroño, España: Universidad de La Rioja.

Romero, F. (2013). Variables financieras determinantes del fracaso empresarial para la pequeña y mediana empresa en Colombia: análisis bajo modelo Logit. Pensamiento \& Gestión, 34, 235-277.

Stanworth, J. and Purdy, D. (1992). Franchise Growth and Failure in the USA and the UK: A 
Troubled Dream World Revisited. Franchising Research: An International Journal, 2, 95-112.

Stanworth, J., Purdy, D. and Price, S. (1997). Franchise growth and failure in the USA and the UK: A troubled dream world revisited. Franchising Research: An InternationalJournal, 2(2), 64-74.

Weaven, S. et al. (2018). Predicting organizational form choice from pre-entry characteristics of franchisees. Australasian Marketing Journal, 26(1), 49-58.

World Bank. (2015). Doing business 2015: going beyond efficiency. Washington, USA: World Bank Group.

WFC. (2013). Franchising: Promoting entrepreneurship and spreading opportunities and wealth. Beirut, Lebanon: Lebanese Franchise Association.

Young, J.and McIntyre, F. (2011). The International Society of Franchising: A Review of Conference Papers Across its First 25 Years. En Kaufmann, P., Lafontaine, F. and Dant, R. (Eds.), Proceedings of the 25th Annual International Society of Franchising Conference. Florida, USA: ISOF, Nova Southeastern University. 